\title{
Active myofascial trigger points in head and neck muscles of patients with chronic tension-type headache in two primary health care units in Tshwane
}

\author{
K Joseph ${ }^{a *}$, SA Hitchcock ${ }^{a}$, HP Meyer ${ }^{a}$, MM Geyser $^{b}$ iD and PJ Becker \\ ${ }^{a}$ Department of Family Medicine, Kalafong Hospital and University of Pretoria, Pretoria, South Africa \\ ${ }^{b}$ Department of Family Medicine and Emergency Medicine, Kalafong Hospital and University of Pretoria, Pretoria, South Africa \\ 'Faculty of Health Sciences, University of Pretoria and Biostatistics Unit, MRC (Pretoria), Pretoria, South Africa \\ *Corresponding author, email: raju@webmail.co.za
}

Abstract (Full text available online at www.tandfonline.com/ojfp)

S Afr Fam Pract 2016; DOI:10.1080/20786190.2015.1120932

Background: The management of patients presenting with chronic tension-type headache (CTTH) can be challenging for primary health care practitioners. As with most chronic pain disorders, a multimodal management approach is frequently required. It has been postulated that myofascial pain syndrome (MPS) and its hallmark myofascial trigger points (MTrPs) found in specific muscle tissues may play a role in the chronic pain experienced by patients with CTTH. Little is known about the prevalence of MTrPs in patients with $\mathrm{CTTH}$, in primary health care settings on the African continent. This study therefore aimed to investigate the prevalence of active MTrP's in specific head and neck muscles/muscle groups in patients with CTTH.

Methods: A prospective, cross-sectional and descriptive study was done in two primary health care facilities situated in Tshwane, South Africa. The sample included 97 adult patients with CTTH. Five head and neck muscles/muscle groups were examined bilaterally for active MTrPs. Outcome measures were the prevalence and distribution of active MTrPs in these patients.

Results: Active MTrPs were found in $95.9 \%$ of the patients, the majority $(74.2 \%)$ having four or more active MTrPs. The temporalis muscles and suboccipital muscle group exhibited the highest number of active MTrPs (prevalence $87.6 \%$ and $80.4 \%$ respectively). Conclusion: Our study suggests a strong association between MPS and CTTH in patients, presenting in the primary health care setting. This indicates the importance of a musculoskeletal assessment of neck and pericranial muscles in patients with CTTH. This can assist in determining the most appropriate treatment strategy in these patients.

Keywords: chronic pain disorders, chronic tension type headache (CTTH), myofascial pain syndrome (MPS), myofascial trigger points (MTrPs), primary headache

\section{Investigation of the coexistence of CKD and non-communicable chronic diseases in a PBM company in South Africa}

\author{
WP Meuwesena, JM du Plessisa*, JR Burgera, MS Lubbe ${ }^{a}$ and M Cockeran ${ }^{a}$ iD \\ ${ }^{a}$ Medicine Usage in South Africa (MUSA), North-West University, Potchefstroom, South Africa \\ *Corresponding author, email: jesslee.duplessis@nwu.ac.za
}

Abstract (Full text available online at www.tandfonline.com/ojfp)

S Afr Fam Pract 2016; DOI:10.1080/20786190.2016.1151641

Background: Chronic kidney disease (CKD) is a public health problem, with increasing global prevalence. Several factors could influence the prognosis of CKD, including comorbid chronic conditions. This study investigated the coexistence of CKD and noncommunicable chronic diseases in the private health sector of South Africa.

Methods: Retrospective medicine claims data from a pharmaceutical benefit management (PBM) company was used to perform this descriptive, quantitative study. The study population consisted of all patients identified with an ICD-10 code for CKD (N18) during the study period of January 1, 2009 to December 31, 2013.

Results: CKD patients represented $0.10 \%$ to $0.14 \%$ of the total patients on the database from 2009 to 2013 . The mean age of the CKD patients over the study period varied between 58 and 61 years. Prevalence was higher in males (male-to-female ratio 1:0.8) and in patients aged 35-64 years ( $p=0.014$; Cramer's $V=0.039$ ). The occurrence of chronic conditions in the CKD population was prevalent, with hypertension occurring in more than half the CKD patients.

Conclusion: Several chronic conditions, especially those regarding atherosclerotic risk factors, frequently co-occurred with CKD. Lifestyle management and frequent screening tests of these patients are of the utmost importance to improve the outcome of CKD.

Keywords: chronic kidney disease, comorbid chronic conditions, risk factors, South Africa 